\title{
An Accurate Block Solver for Stiff Initial Value Problems
}

\author{
H. Musa, ${ }^{1}$ M. B. Suleiman, ${ }^{2}$ F. Ismail, ${ }^{1}$ N. Senu, ${ }^{1}$ and Z. B. Ibrahim ${ }^{1}$ \\ ${ }^{1}$ Department of Mathematics, Faculty of Science, Universiti Putra Malaysia, 43400 Serdang, Selangor, Malaysia \\ ${ }^{2}$ Institute for Mathematical Research, Universiti Putra Malaysia, 43400 Serdang, Selangor, Malaysia \\ Correspondence should be addressed to M. B. Suleiman; mohameds@science.upm.edu.my
}

Received 19 June 2013; Accepted 20 July 2013

Academic Editors: S. Blanes and L. You

Copyright @ $2013 \mathrm{H}$. Musa et al. This is an open access article distributed under the Creative Commons Attribution License, which permits unrestricted use, distribution, and reproduction in any medium, provided the original work is properly cited.

\begin{abstract}
New implicit block formulae that compute solution of stiff initial value problems at two points simultaneously are derived and implemented in a variable step size mode. The strategy for changing the step size for optimum performance involves halving, increasing by a multiple of 1.7, or maintaining the current step size. The stability analysis of the methods indicates their suitability for solving stiff problems. Numerical results are given and compared with some existing backward differentiation formula algorithms. The results indicate an improvement in terms of accuracy.
\end{abstract}

\section{Introduction}

Stiff problems occur in many fields of engineering science and they represent coupled physical systems having components varying with very different time scales [1]. Some physical examples include chemical kinetics where reactions go at very different speeds and in circuit simulation where components respond at widely different time scales. A considerable research has been done on numerical methods for solving stiff initial value problems (IVPs) of the following form:

$$
y^{\prime}=f(x, y) \quad y\left(x_{0}\right)=y_{0}, \quad x \in[a, b] .
$$

The most successful ones are implicit in nature. Examples include single step methods like the diagonally implicit Runge Kutta (DIRK) methods, single diagonally implicit RungeKutta (SDIRK), and singly implicit Runge-Kutta methods [2-4]. Also well known are methods based on Richardson extrapolation and deferred correction schemes [5-8]. Others are multistep methods like the backward differentiation formula (BDF) (see, e.g., [4]). Initially, the BDF methods are known to be $A$-stable only up to order 2 [9]. However, Cash in [10] proved that the barrier in [9] can be circumvented by adding extra future point to produce higher order $A$-stable methods. Methods of this type are known as extended backward differentiation formula [10-12]. Some block methods for stiff problems are also known to be $A$-stable and have order greater than two (see, e.g., [13-18]).

One of the motivation of this paper is to develop a new block method based on the block backward differentiation formula $[13,14,17]$. The method is similar in fashion with that in [15] but differs greatly by the choice of the constant $\rho$. Furthermore, our choice of $\rho=3 / 8$ gives rise to different formulae with better stability regions than in [15], even with the choice of the same step ratio $r$. In the following sections of the paper, we will present the derivation of the method, its stability analysis, and implementation. Standard test problems will also be presented and a comparison of their numerical results with some known stiff solvers will be given.

\section{Formulation of the Method}

Consider a special case of the following BBDF given in [14, 17]:

$$
\sum_{j=0}^{4} \alpha_{j, i, r} y_{n+j-2}=h \beta_{k, i, r} f_{n+k}, \quad k=i=1,2,
$$

where $h=$ the variable step size used; $x_{n}=a+n h ; y_{n}=$ computed solution; $f_{n}=f\left(x_{n}, y_{n}\right), n \geq 0 ; \beta_{0, i, r}=\beta_{1, i, r}=$ $\cdots=\beta_{k-1, i, r}=0 ; k=i=1$ represents the formula for the first point; $k=i=2$ represents the formula for the second point; and $r=$ the value used in the step size changing strategy. 
In this paper, a nonzero coefficient $\beta_{k-1, i, r}=\rho \beta_{k, i, r}$ is introduced in (2) to have a formula of the following form:

$$
\sum_{j=0}^{4} \alpha_{j, i, r} y_{n+j-2}=h \beta_{k, i, r}\left(f_{n+k}-\rho f_{n+k-1}\right), \quad k=i=1,2,
$$

where $\beta_{k-1, i, r}=\rho \beta_{k, i, r}$.

Define the linear difference operator $L_{i}$ associated with the method (3) by

$$
\begin{aligned}
L_{i}\left(y\left(x_{n}\right), h\right)= & \alpha_{0, i} y\left(x_{n}-2 r h\right)+\alpha_{1, i} y\left(x_{n}-r h\right) \\
& +\alpha_{2, i} y\left(x_{n}\right)+\alpha_{3, i} y\left(x_{n}+h\right) \\
& +\alpha_{4, i} y\left(x_{n}+2 h\right) \\
& -h \beta_{k, i}\left(f\left(x_{n}+k h\right)-\rho f\left(x_{n}+(k-1) h\right)\right) \\
= & 0,
\end{aligned}
$$

where $k=i=1$ stands for the first point and $k=i=2$ stands for the second point. Expanding (4) using Taylor's series and collecting like terms give a set of equations to be solved for $\alpha_{j, i^{\prime} s}$ and $\beta_{k, i^{\prime} s}$. For the derivation of the first point, $\alpha_{3,1}$ is normalized to 1 and for the second point, $\alpha_{4,2}$ is normalized to 1 ; the following 2-point implicit block method is obtained as

$$
\begin{aligned}
& y_{n+1}=\frac{-1-r-2 \rho r}{4 r^{2}\left(-2-3 r+4 \rho r^{2}\right)} y_{n-2} \\
& +\frac{1+4 r+4 \rho r+4 r^{2}+8 \rho r^{2}}{r^{2}(2+r)\left(-2-3 r+4 \rho r^{2}\right)} y_{n-1} \\
& +\frac{-1-6 r-6 \rho r-13 r^{2}-12 \rho r^{2}-12 r^{3}+6 \rho r^{3}-4 r^{4}+12 \rho r^{4}}{4 r^{2}\left(-2-3 r+4 \rho r^{2}\right)} y_{n} \\
& +\frac{1+5 r+8 r^{2}+2 \rho r^{2}+4 r^{3}+4 \rho r^{3}}{4(2+r)\left(-2-3 r+4 \rho r^{2}\right)} y_{n+2} \\
& +\frac{1+3 r+2 r^{2}}{-2-3 r+4 \rho r^{2}} \rho h f_{n} \\
& -\frac{1+3 r+2 r^{2}}{-2-3 r+4 \rho r^{2}} h f_{n+1}, \\
& y_{n+2}=\frac{8+2 \rho+8 r+3 \rho r+2 r^{2}+\rho r^{2}}{r^{2}(1+2 r)\left(-20+\rho-24 r+3 \rho r-6 r^{2}+2 \rho r^{2}\right)} y_{n-2} \\
& -\frac{4(4+\rho+4 r+2 \rho r)}{r^{2}\left(-20+\rho-24 r+3 \rho r-6 r^{2}+2 \rho r^{2}\right)} y_{n-1} \\
& +\frac{8+2 \rho+24 r+9 \rho r+26 r^{2}+14 \rho r^{2}+12 r^{3}+9 \rho r^{3}+2 r^{4}+2 \rho r^{4}}{r^{2}\left(-20+\rho-24 r+3 \rho r-6 r^{2}+2 \rho r^{2}\right)} y_{n} \\
& -\frac{4\left(16+4 \rho+32 r+8 \rho r+20 r^{2}+3 \rho r^{2}+4 r^{3}\right)}{(1+2 r)\left(-20+\rho-24 r+3 \rho r-6 r^{2}+2 \rho r^{2}\right)} y_{n+1} \\
& +\frac{4\left(2+3 r+r^{2}\right)}{-20+\rho-24 r+3 \rho r-6 r^{2}+2 \rho r^{2}} h \rho f_{n+1} \\
& -\frac{4\left(2+3 r+r^{2}\right)}{-20+\rho-24 r+3 \rho r-6 r^{2}+2 \rho r^{2}} h f_{n+2} .
\end{aligned}
$$

For optimal performance, we choose different parameters from those used in [15]. We choose $\rho=3 / 8$ and $r=1,2$ and $10 / 17$ where the values of $r$ correspond to the step size control strategy of maintaining constant, halving, and increasing the step size by a multiple of 1.7.

When $r=1$ we have

$$
\begin{aligned}
y_{n+1}= & \frac{11}{56} y_{n-2}-\frac{9}{7} y_{n-1}+\frac{18}{7} y_{n} \\
& -\frac{27}{56} y_{n+2}-\frac{9}{14} h f_{n}+\frac{12}{7} h f_{n+1}, \\
y_{n+2}= & -\frac{27}{191} y_{n-2}+\frac{146}{191} y_{n-1}-\frac{342}{191} y_{n} \\
& +\frac{414}{191} y_{n+1}-\frac{36}{191} h f_{n+1}+\frac{96}{191} h f_{n+2} .
\end{aligned}
$$

When $r=2$ we have

$$
\begin{aligned}
y_{n+1}= & \frac{9}{64} y_{n-2}-\frac{5}{4} y_{n-1}+\frac{315}{64} y_{n} \\
& -\frac{45}{16} y_{n+2}-\frac{45}{16} h f_{n}+\frac{15}{2} h f_{n+1}, \\
y_{n+2}= & -\frac{73}{3455} y_{n-2}+\frac{111}{691} y_{n-1}-\frac{711}{691} y_{n} \\
& +\frac{6528}{3455} y_{n+1}-\frac{144}{691} h f_{n+1}+\frac{384}{691} h f_{n+2} .
\end{aligned}
$$

And when $r=10 / 17$ we have

$$
\begin{aligned}
y_{n+1}= & \frac{338997}{750400} y_{n-2}-\frac{2363153}{1031800} y_{n-1}+\frac{2310687}{750400} y_{n} \\
& -\frac{19869}{82544} y_{n+2}-\frac{2997}{7504} h f_{n}+\frac{999}{938} h f_{n+1}, \\
y_{n+2}= & -\frac{8484751}{14926355} y_{n-2}+\frac{191607}{80683} y_{n-1} \\
& -\frac{1307097}{403415} y_{n}+\frac{7265280}{2985271} y_{n+1} \\
& -\frac{14256}{80683} h f_{n+1}+\frac{38016}{80683} h f_{n+2} .
\end{aligned}
$$

Formulae (6), (7), and (8) compute two solution values concurrently at each step of the integration thereby having the advantage of reducing the total number of steps when a conventional nonblock method is used. Also, the formulae obtained are entirely different from those in [15]. It should be noted that when $\rho=0$ in (5), formula (3) reduces to (2) and we say that formula (2) is contained in (3). Hence, (3) is a superclass of (2) and contains (2) as a subclass. We will refer to our method as variable step size superclass of block backward differentiation formula (VSSBBDF). In the following section, the stability analysis of the method will be presented. 


\section{Stability of the Method}

This section investigates the stability regions of the methods (6), (7), and (8). When $r=1$, the formulae in (6) can be represented as:

$$
\begin{aligned}
\left(\begin{array}{ll}
1 & 0 \\
0 & 1
\end{array}\right)\left(\begin{array}{l}
y_{n+1} \\
y_{n+2}
\end{array}\right)= & \left(\begin{array}{cc}
0 & \frac{11}{56} \\
0 & -\frac{27}{191}
\end{array}\right)\left(\begin{array}{l}
y_{n-3} \\
y_{n-2}
\end{array}\right) \\
& +\left(\begin{array}{cc}
-\frac{9}{7} & \frac{18}{7} \\
\frac{146}{191} & -\frac{342}{191}
\end{array}\right)\left(\begin{array}{c}
y_{n-1} \\
y_{n}
\end{array}\right) \\
& +\left(\begin{array}{cc}
0 & -\frac{27}{56} \\
\frac{414}{191} & 0
\end{array}\right)\left(\begin{array}{c}
y_{n+1} \\
y_{n+2}
\end{array}\right) \\
& +h\left(\begin{array}{cc}
0 & -\frac{9}{14} \\
0 & 0
\end{array}\right)\left(\begin{array}{c}
f_{n-1} \\
f_{n}
\end{array}\right) \\
& +h\left(\begin{array}{cc}
\frac{12}{7} & 0 \\
-\frac{36}{191} & \frac{96}{191}
\end{array}\right)\left(\begin{array}{l}
f_{n+1} \\
f_{n+2}
\end{array}\right) .
\end{aligned}
$$

Collecting like terms we have

$$
\begin{aligned}
\left(\begin{array}{cc}
1 & \frac{27}{56} \\
-\frac{414}{191} & 1
\end{array}\right)\left(\begin{array}{l}
y_{n+1} \\
y_{n+2}
\end{array}\right)= & \left(\begin{array}{cc}
0 & \frac{11}{56} \\
0 & -\frac{27}{191}
\end{array}\right)\left(\begin{array}{l}
y_{n-3} \\
y_{n-2}
\end{array}\right) \\
& +\left(\begin{array}{cc}
-\frac{9}{7} & \frac{18}{7} \\
\frac{146}{191} & -\frac{342}{191}
\end{array}\right)\left(\begin{array}{c}
y_{n-1} \\
y_{n}
\end{array}\right) \\
& +h\left(\begin{array}{cc}
0 & -\frac{9}{14} \\
0 & 0
\end{array}\right)\left(\begin{array}{c}
f_{n-1} \\
f_{n}
\end{array}\right) \\
& +h\left(\begin{array}{cc}
\frac{12}{7} & 0 \\
-\frac{36}{191} & \frac{96}{191}
\end{array}\right)\left(\begin{array}{l}
f_{n+1} \\
f_{n+2}
\end{array}\right) .
\end{aligned}
$$

Let $h \lambda=\bar{h}$. Applying the scalar test equation,

$$
y^{\prime}=\lambda y \quad \lambda<0,
$$

to $(10)$ gives

$$
\begin{gathered}
\left(\begin{array}{cc}
1-\frac{12}{7} \bar{h} & \frac{27}{56} \\
-\frac{414}{191}+\frac{36}{191} \bar{h} & 1-\frac{96}{191} \bar{h}
\end{array}\right)\left(\begin{array}{l}
y_{n+1} \\
y_{n+2}
\end{array}\right) \\
=\left(\begin{array}{cc}
-\frac{9}{7} & \frac{18}{7}-\frac{9}{14} \bar{h} \\
\frac{146}{191} & -\frac{342}{191}
\end{array}\right)\left(\begin{array}{c}
y_{n-1} \\
y_{n}
\end{array}\right) \\
+\left(\begin{array}{cc}
0 & \frac{11}{56} \\
0 & -\frac{27}{191}
\end{array}\right)\left(\begin{array}{l}
y_{n-3} \\
y_{n-2}
\end{array}\right) .
\end{gathered}
$$

Equation (12) is equivalent to

$$
A Y_{m}=B Y_{m-1}+C Y_{m-2}, \quad n=2 m,
$$

where

$$
\begin{gathered}
A=\left(\begin{array}{cc}
1-\frac{12}{7} \bar{h} & \frac{27}{56} \\
-\frac{414}{191}+\frac{36}{191} \bar{h} & 1-\frac{96}{191} \bar{h}
\end{array}\right), \\
B=\left(\begin{array}{ccc}
-\frac{9}{7} & \frac{18}{7}-\frac{9}{14} \bar{h} \\
\frac{146}{191} & -\frac{342}{191}
\end{array}\right), \\
C=\left(\begin{array}{cc}
0 & \frac{11}{56} \\
0 & -\frac{27}{191}
\end{array}\right), \quad Y_{m}=\left(\begin{array}{l}
y_{n+1} \\
y_{n+2}
\end{array}\right)=\left(\begin{array}{l}
y_{2 m+1} \\
y_{2 m+2}
\end{array}\right), \\
Y_{m-1}=\left(\begin{array}{c}
y_{n-1} \\
y_{n}
\end{array}\right)=\left(\begin{array}{c}
y_{2 m-1} \\
y_{2 m}
\end{array}\right)=\left(\begin{array}{c}
y_{2(m-1)+1} \\
y_{2(m-1)+2}
\end{array}\right), \\
Y_{m-2}=\left(\begin{array}{c}
y_{n-3} \\
y_{n-2}
\end{array}\right)=\left(\begin{array}{l}
y_{2(m-2)+1} \\
y_{2(m-2)+2}
\end{array}\right) .
\end{gathered}
$$

The stability polynomial $R(t, \bar{h})$ is obtained by evaluating the determinant of $\left(A t^{2}-B t-C\right)$ to obtain

$$
\begin{aligned}
R(t, \bar{h})= & \frac{169 t}{5348}+\frac{279 t^{2}}{5348}+\frac{765 \bar{h} t^{2}}{2674} \\
& -\frac{11385 t^{3}}{5348}-\frac{351 \bar{h} t^{3}}{191}-\frac{162 \bar{h}^{2} t^{3}}{1337} \\
& +\frac{10937 t^{4}}{5348}-\frac{6171 \bar{h} t^{4}}{2674}+\frac{1152 \bar{h}^{2} t^{4}}{1337} \\
= & 0 .
\end{aligned}
$$

To show that the method is zero stable, we let $\bar{h}=0$ in (15) and the following equation for the first characteristics polynomial is obtained as

$$
\frac{169 t}{5348}+\frac{279 t^{2}}{5348}-\frac{11385 t^{3}}{5348}+\frac{10937 t^{4}}{5348}=0 .
$$


Solving (16) for $t$, we have

$$
\begin{gathered}
t=0, \quad t=1, \quad t=-0.105502, \\
t=0.146463
\end{gathered}
$$

The values of $t$ above indicate that the method is zero stable since no magnitude of the root is greater than one and the root $t=1$ is simple.

The plot of the stability polynomial (15) is given in Figure 1. The region of stability is the region outside the circular shape. It indicates that the method (6) is $A$-stable since the region covers the entire negative half plane.

The same procedure is applied to obtain the stability polynomial of the method (7) (corresponding to $r=2$ ) as

$$
\begin{aligned}
R(t, \bar{h})= & \frac{169 t}{44224}+\frac{11099 t^{2}}{44224}+\frac{3567 \bar{h} t^{2}}{11056} \\
& -\frac{72625 t^{3}}{11056}-\frac{5727 \bar{h} t^{3}}{2764}-\frac{405 \bar{h}^{2} t^{3}}{691} \\
& +\frac{4363 t^{4}}{691}-\frac{11943 \bar{h} t^{4}}{1382}+\frac{2880 \bar{h}^{2} t^{4}}{691} \\
= & 0 .
\end{aligned}
$$

When $\bar{h}=0$ in (18), the first characteristic polynomial of the method (7) becomes

$$
\frac{169 t}{44224}+\frac{11099 t^{2}}{44224}-\frac{72625 t^{3}}{11056}+\frac{4363 t^{4}}{691}=0
$$

and solving (19) for $t$ gives the following roots:

$$
\begin{gathered}
t=0, \quad t=1, \quad t=-0.0116404, \\
t=0.051994
\end{gathered}
$$

Again, it shows that the method (7) is zero stable.

The stability plot of the polynomial (18) is given in Figure 2 and the region outside the circle indicates that the method is also $A$-stable.

Similarly, the stability polynomial for the method (8) (corresponding to $r=10 / 17$ ) is given by

$$
\begin{aligned}
R(t, \bar{h})= & \frac{69347235737 t}{302722616000}-\frac{128013466997 t^{2}}{302722616000} \\
& +\frac{6400808703 \bar{h} t^{2}}{15136130800}-\frac{21069763237 t^{3}}{15136130800} \\
& -\frac{1629222471 \bar{h} t^{3}}{540576100}-\frac{2670327 \bar{h}^{2} t^{3}}{37840327} \\
& +\frac{60007687 t^{4}}{37840327}-\frac{119480103 \bar{h} t^{4}}{75680654} \\
& +\frac{18988992 \bar{h}^{2} t^{4}}{37840327} \\
= & 0
\end{aligned}
$$

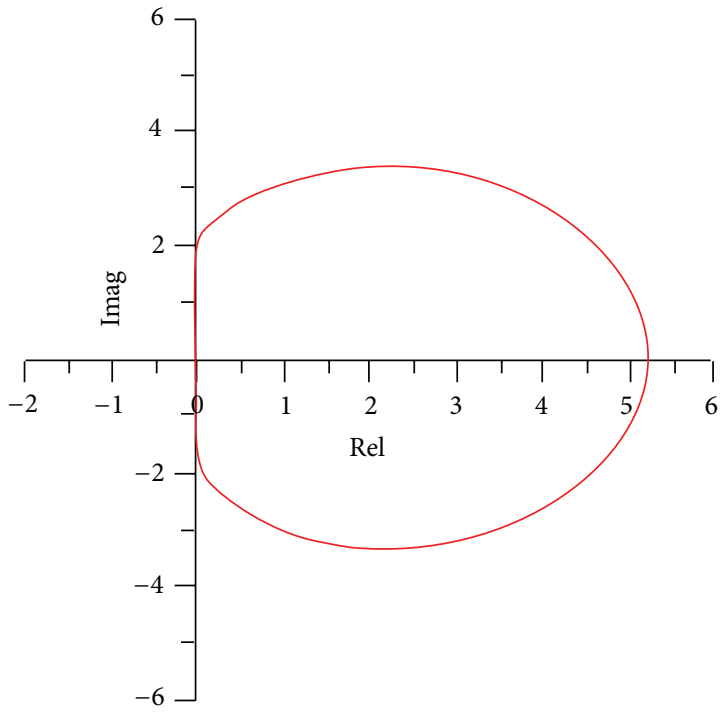

FIGURE 1: Stability region of the 2-point VSSBBDF when $r=1$.

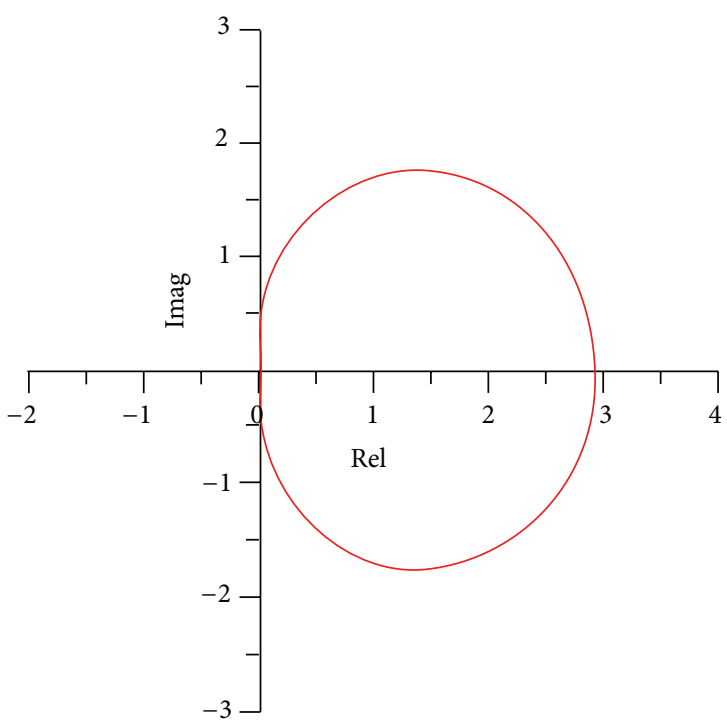

FIgURE 2: Stability region of the 2-point VSSBBDF when $r=2$.

and when $\bar{h}=0$ in (21), the first characteristics polynomial is given by

$$
\begin{aligned}
& \frac{69347235737 t}{302722616000}-\frac{128013466997 t^{2}}{302722616000} \\
& \quad-\frac{21069763237 t^{3}}{15136130800}+\frac{60007687 t^{4}}{37840327} \\
& =0 .
\end{aligned}
$$

When (22) is solved for $t$, the following values are obtained:

$$
\begin{gathered}
t=0, \quad t=1, \quad t=-0.446055, \\
t=0.32385
\end{gathered}
$$

Hence, the method (8) is also zero stable. 
The stability plot for (21) is given in Figure 3. The method (8) is therefore almost $A$-stable.

$A$-stability is a desirable property for numerical methods of solving stiff problems. From the forgoing analysis, the methods developed are suitable for solving stiff initial value problems. In the following section, the procedure involved in the implementation of the method will be explained.

\section{Implementation of the Method}

The implementation involves Newton's iteration. To simplify the code, methods (6), (7), and (8) are written in the following form:

$$
\begin{aligned}
& y_{n+1}=\theta_{1} y_{n+2}+\alpha_{1} h f_{n}+\alpha_{2} h f_{n+1}+\Psi_{1}, \\
& y_{n+2}=\theta_{2} y_{n+1}+\alpha_{3} h f_{n+1}+\alpha_{4} h f_{n+2}+\Psi_{2},
\end{aligned}
$$

which is equivalent to the following matrix equation:

$$
\begin{aligned}
\left(\begin{array}{ll}
1 & 0 \\
0 & 1
\end{array}\right)\left(\begin{array}{l}
y_{n+1} \\
y_{n+2}
\end{array}\right)= & \left(\begin{array}{cc}
0 & \theta_{1} \\
\theta_{2} & 0
\end{array}\right)\left(\begin{array}{l}
y_{n+1} \\
y_{n+2}
\end{array}\right) \\
& +h\left(\begin{array}{cc}
0 & \alpha_{1} \\
0 & 0
\end{array}\right)\left(\begin{array}{c}
f_{n-1} \\
f_{n}
\end{array}\right) \\
& +h\left(\begin{array}{cc}
\alpha_{2} & 0 \\
\alpha_{3} & \alpha_{4}
\end{array}\right)\left(\begin{array}{l}
f_{n+1} \\
f_{n+2}
\end{array}\right)+\left(\begin{array}{l}
\Psi_{1} \\
\Psi_{2}
\end{array}\right) .
\end{aligned}
$$

Equation (25) can be represented as

$$
(I-A) Y=h\left(B_{1} F_{1}+B_{2} F_{2}\right)+\zeta,
$$

where

$$
\begin{gathered}
I=\left(\begin{array}{ll}
1 & 0 \\
0 & 1
\end{array}\right), \quad A=\left(\begin{array}{cc}
0 & \theta_{1} \\
\theta_{2} & 0
\end{array}\right), \quad B_{1}=\left(\begin{array}{cc}
0 & \alpha_{1} \\
0 & 0
\end{array}\right), \\
B_{2}=\left(\begin{array}{cc}
\alpha_{2} & 0 \\
\alpha_{3} & \alpha_{4}
\end{array}\right), \quad Y=\left(\begin{array}{c}
y_{n+1} \\
y_{n+2}
\end{array}\right), \quad F_{1}=\left(\begin{array}{c}
f_{n-1} \\
f_{n}
\end{array}\right), \\
F_{2}=\left(\begin{array}{c}
f_{n+1} \\
f_{n+2}
\end{array}\right), \quad \zeta=\left(\begin{array}{l}
\Psi_{1} \\
\Psi_{2}
\end{array}\right) .
\end{gathered}
$$

Let

$$
F=(I-A) Y-h\left(B_{1} F_{1}+B_{2} F_{2}\right)-\zeta=0 .
$$

Newton's iteration takes the form

$$
Y_{n+1, n+2}^{(i+1)}-Y_{n+1, n+2}^{(i)}=-\left[F_{j}^{\prime}\left(y_{n+1, n+2}^{(i)}\right)\right]^{-1}\left[F_{j}\left(y_{n+1, n+2}^{(i)}\right)\right] .
$$

Equation (32) is equivalent to

$$
\begin{aligned}
Y_{n+1, n+2}^{(i+1)} & -Y_{n+1, n+2}^{(i)} \\
= & -\left[(I-A)-h B_{1} \frac{\delta F_{1}}{\delta Y}\left(Y_{n+1, n+2}^{(i)}\right)-h B_{2} \frac{\delta F_{2}}{\delta Y}\left(Y_{n+1, n+2}^{(i)}\right)\right]^{-1} \\
& \times\left[(I-A)\left(Y_{n+1, n+2}^{(i)}\right)-h B_{1} F_{1}-h B_{2} F_{2}-\zeta\right] .
\end{aligned}
$$

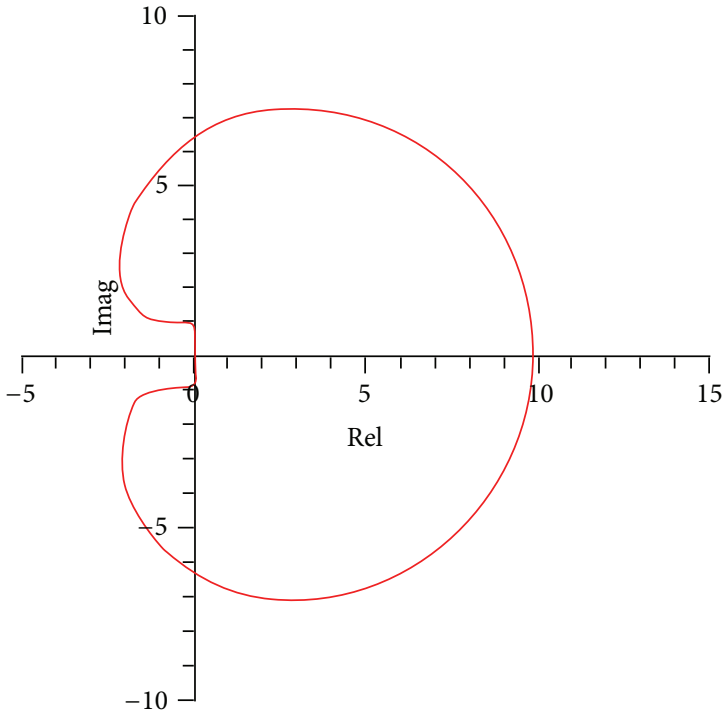

FIGURE 3: Stability region of the 2-point VSSBBDF when $r=10 / 17$.

We show the accuracy of the method by finding the maximum error in the following way. Let $y_{i}$ and $y\left(x_{i}\right)$ be the computed and the exact solutions of (1), respectively. The absolute error is defined by

$$
\left(\text { error }_{i}\right)_{t}=\left|\left(y_{i}\right)_{t}-\left(y\left(x_{i}\right)\right)_{t}\right| \text {. }
$$

The maximum error is given by:

$$
\text { MAXE }=\underbrace{\max }_{1 \leq t \leq T}(\underbrace{\max }_{1 \leq i \leq N}\left(\text { error }_{i}\right)_{t}),
$$

where $T$ is the total number of steps and $N$ is the number of equations.

Let $Y_{n+1}^{(i+1)}$ denote the $(i+1)$ th iterate and

$$
E_{1,2}^{(i+1)}=Y_{n+1, n+2}^{(i+1)}-Y_{n+1, n+2}^{(i)} .
$$

Then (33) can be written as

$$
E_{1,2}^{(i+1)}=\bar{A}^{-1} \bar{B}
$$

which is equivalent to

$$
\bar{A} E_{1,2}^{(i+1)}=\bar{B}
$$

where

$$
\begin{gathered}
\bar{A}=\left[(I-A)-h B_{1} \frac{\delta F_{1}}{\delta Y}\left(Y_{n+1, n+2}^{(i)}\right)-h B_{2} \frac{\delta F_{2}}{\delta Y}\left(Y_{n+1, n+2}^{(i)}\right)\right]^{-1}, \\
\bar{B}=-\left[(I-A)\left(Y_{n+1, n+2}^{(i)}\right)-h B_{1} F_{1}-h B_{2} F_{2}-\zeta\right] .
\end{gathered}
$$

LU decomposition method is used to solve the system (35). For the different values of $r$,

$$
\bar{A}=\left(\begin{array}{cc}
1-\alpha_{2} h \frac{\delta f_{n+1}}{\delta y_{n+1}} & -\theta_{1} \\
-\theta_{2}-\alpha_{3} h \frac{\delta f_{n+1}}{\delta y_{n+1}} & 1-\alpha_{4} h \delta \frac{f_{n+2}}{\delta y_{n+2}}
\end{array}\right),
$$




$$
\bar{B}=\left(\begin{array}{c}
-y_{n+1}^{i}+\theta_{1} y_{n+2}^{i}+\alpha_{1} h f_{n}^{i}+\alpha_{2} h f_{n+1}^{i}+\Psi \\
-y_{n+2}^{i}+\theta_{2} y_{n+1}^{i}+\alpha_{3} h f_{n+1}^{i}+\alpha_{4} h f_{n+2}^{i}+\Psi
\end{array}\right) . \quad \text { When } r=1
$$

$$
\begin{gathered}
\bar{A}=\left(\begin{array}{c}
1-\frac{12}{7} h \frac{\delta f_{n+1}}{\delta y_{n+1}} \\
-\frac{414}{191}+\frac{36}{191} h \frac{\delta f_{n+1}}{\delta y_{n+1}} 1-\frac{96}{191} h \delta \frac{f_{n+2}}{\delta y_{n+2}}
\end{array}\right), \\
\bar{B}=\left(\begin{array}{c}
-y_{n+1}^{i}-\frac{27}{56} y_{n+2}^{i}-\frac{9}{14} h f_{n}^{i}+\frac{12}{7} h f_{n+1}^{i}+\frac{11}{56} y_{n-2}^{i}-\frac{9}{7} y_{n-1}+\frac{18}{7} y_{n} \\
-y_{n+2}^{i}+\frac{414}{191} y_{n+1}^{i}-\frac{36}{191} h f_{n+1}^{i}+\frac{96}{191} h f_{n+2}^{i}-\frac{27}{191} y_{n-2}+\frac{146}{191} y_{n-1}-\frac{342}{191} y_{n}
\end{array}\right) .
\end{gathered}
$$

When $r=2$

$$
\begin{gathered}
\bar{A}=\left(\begin{array}{c}
1-\frac{15}{2} h \frac{\delta f_{n+1}}{\delta y_{n+1}} \\
-\frac{6528}{3455}+\frac{144}{691} h \frac{\delta f_{n+1}}{\delta y_{n+1}} 1-\frac{384}{691} h \delta \frac{f_{n+2}}{\delta y_{n+2}}
\end{array}\right), \\
\bar{B}=\left(\begin{array}{c}
-y_{n+1}^{i}-\frac{45}{16} y_{n+2}^{i}-\frac{45}{16} h f_{n}^{i}+\frac{15}{2} h f_{n+1}^{i}+\frac{9}{64} y_{n-2}-\frac{5}{4} y_{n-1}+\frac{315}{64} y_{n} \\
-y_{n+2}^{i}+\frac{6528}{3455} y_{n+1}^{i}-\frac{144}{691} h f_{n+1}^{i}+\frac{384}{691} h f_{n+2}^{i}-\frac{73}{3455} y_{n-2}+\frac{111}{691} y_{n-1}-\frac{711}{691} y_{n}
\end{array}\right)
\end{gathered}
$$

and when $r=10 / 17$

$$
\begin{gathered}
\bar{A}=\left(\begin{array}{c}
1-\frac{999}{938} h \frac{\delta f_{n+1}}{\delta y_{n+1}} \\
-\frac{7265280}{2985271}+\frac{14256}{80683} h \frac{\delta f_{n+1}}{\delta y_{n+1}} 1-\frac{38016}{80683} h \delta \frac{f_{n+2}}{\delta y_{n+2}}
\end{array}\right), \\
\bar{B}=\left(\begin{array}{c}
-y_{n+1}^{i}-\frac{19869}{82544} y_{n+2}^{i}-\frac{2997}{7504} h f_{n}^{i}+\frac{999}{938} h f_{n+1}^{i}+\frac{338997}{750400} y_{n-2}-\frac{2363153}{1031800} y_{n-1}+\frac{2310687}{750400} y_{n} \\
-y_{n+2}^{i}+\frac{7265280}{2985271} y_{n+1}^{i}-\frac{14256}{80683} h f_{n+1}^{i}+\frac{38016}{80683} h f_{n+2}^{i}-\frac{8484751}{14926355} y_{n-2}+\frac{191607}{80683} y_{n-1}-\frac{1307097}{403415} y_{n}
\end{array}\right) .
\end{gathered}
$$

4.1. Step Size Selection. The step size increase in this paper differs with that in [15] in the sense that an increase is made by a multiple of 1.7 of the previous step size. This is done in order to optimize total number of steps and computation time. Three techniques are employed in controlling the step size. At the beginning of the integration, an initial step size is determined and the local truncation error (LTE) is computed as follows:

$$
\mathrm{LTE}=\left|y_{n+2}^{k+1}-y_{n+2}^{k}\right|, \quad k=3,
$$

where $y_{n+2}^{k+1}$ refers to the formula of order $k+1$ and $y_{n+2}^{k}$ refers to the formula of order $k$.

If the LTE is less than or equal to a specified error tolerance (TOL) (i.e., LTE $\leq$ TOL), the step size (from the previous block) is maintained as constant (equivalent to the formula when $r=1$ ) and a new step size is computed as

$$
{ }_{\text {new }} h=c \times \times_{\text {old }} h \times\left(\frac{\text { TOL }}{\mathrm{LTE}}\right)^{1 / p} \text {, }
$$

where $p$ is the order of the method and $c$ is a safety factor. Again we choose a safety factor $(c=0.3)$ different from that in [15].

If ${ }_{\text {new }} h>\left(1.7 \times_{\text {old }} h\right)$, then the step size $h$ becomes $h=1.7 \times{ }_{\text {old }} h$ (equivalent to the formula when $r=10 / 17$ ). Otherwise if LTE > TOL, the step size is halved (equivalent to the formula when $r=2$ ). 
TABLE 1: Numerical results for problem (1) (the circuit problem).

\begin{tabular}{|c|c|c|c|c|c|}
\hline TOL & Method & IST & FS & TS & MAXE \\
\hline \multirow{4}{*}{$10^{-2}$} & NBDF & 23 & 0 & 23 & $2.74641 e-003$ \\
\hline & VSBBDF & 63 & 0 & 63 & $2.24782 e-005$ \\
\hline & NVSBBDF & 43 & 0 & 43 & $2.76983 e-005$ \\
\hline & VSSBBDF & 44 & 0 & 44 & $2.34920 e-006$ \\
\hline \multirow{4}{*}{$10^{-4}$} & $\mathrm{NBDF}$ & 121 & 0 & 121 & $3.62021 e-005$ \\
\hline & VSBBDF & 77 & 0 & 77 & $2.24754 e-006$ \\
\hline & NVSBBDF & 62 & 0 & 62 & $3.06417 e-007$ \\
\hline & VSSBBDF & 70 & 0 & 70 & $2.73764 e-008$ \\
\hline \multirow{4}{*}{$10^{-6}$} & NBDF & 365 & 0 & 365 & $3.82424 e-007$ \\
\hline & VSBBDF & 107 & 0 & 107 & $2.24755 e-007$ \\
\hline & NVSBBDF & 101 & 0 & 101 & $3.85316 e-009$ \\
\hline & VSSBBDF & 142 & 0 & 142 & $3.45127 e-010$ \\
\hline
\end{tabular}

TABLE 2: Numerical results for problem (2) (the cosine problem).

\begin{tabular}{|c|c|c|c|c|c|}
\hline TOL & Method & IST & FS & TS & MAXE \\
\hline \multirow{4}{*}{$10^{-2}$} & NBDF & 126 & 38 & 164 & $8.46687 e-005$ \\
\hline & VSBBDF & 72 & 4 & 76 & $5.24918 e-005$ \\
\hline & NVSBBDF & 65 & 1 & 66 & $5.16437 e-005$ \\
\hline & VSSBBDF & 72 & 0 & 72 & $1.23612 e-005$ \\
\hline \multirow{4}{*}{$10^{-4}$} & $\mathrm{NBDF}$ & 249 & 70 & 319 & $3.42565 e-006$ \\
\hline & VSBBDF & 159 & 7 & 166 & $2.12255 e-006$ \\
\hline & NVSBBDF & 116 & 0 & 116 & $1.54385 e-006$ \\
\hline & VSSBBDF & 149 & 0 & 149 & $6.74134 e-007$ \\
\hline \multirow{4}{*}{$10^{-6}$} & NBDF & 474 & 100 & 574 & $1.33439 e-007$ \\
\hline & VSBBDF & 457 & 19 & 476 & $4.32393 e-008$ \\
\hline & NVSBBDF & 398 & 4 & 402 & $3.11839 e-008$ \\
\hline & VSSBBDF & 472 & 0 & 472 & $3.19856 e-009$ \\
\hline
\end{tabular}

\section{Test Problems}

The performance of the method is tested on the following stiff problems that are commonly found in engineering and physical sciences.

(1) The circuit problem taken from [17] is as follows:

$$
y^{\prime}=-20 y+24, \quad y(0)=0, \quad 0 \leq x \leq 10 .
$$

The exact solution is as follows:

$$
y(x)=\frac{6}{5}-\frac{6}{5} e^{-20 x} .
$$

(2) The cosine problem taken from $[8,15,19]$ is as follows:

$$
\begin{gathered}
y^{\prime}=-2 \pi \sin (2 \pi x)-\frac{1}{\epsilon}(y-\cos (2 \pi x)), \\
y(0)=1, \quad 0 \leq x \leq 10 .
\end{gathered}
$$

The problem becomes increasingly stiff as $\epsilon \rightarrow 0$. We choose $\epsilon=10^{-3}$.

The exact solution is as follows:

$$
y(x)=\cos (2 \pi x) .
$$

(3) We consider the system of nonlinear stiff IVP taken from $[18,20]$ as follows:

$$
\begin{gathered}
y_{1}^{\prime}=-\left(\epsilon^{-1}+2\right) y_{1}+\epsilon^{-1} y_{2}^{2}, \quad y_{1}(0)=1, \quad 0 \leq x \leq 20 \\
y_{2}^{\prime}=y_{1}-y_{2}\left(1+y_{2}\right), \quad y_{2}(0)=1 .
\end{gathered}
$$

The solution, which is independent of $\epsilon$, is

$$
\begin{aligned}
& y_{1}(x)=e^{-2 x} \\
& y_{2}(x)=e^{-x} .
\end{aligned}
$$

The eigenvalues are $\lambda_{1}=-1$ and $\lambda_{2}=-\left(\epsilon^{-1}+2\right)$. This allows the degree of stiffness to be regulated. We choose $\epsilon=10^{-5}$.

(4) This physical problem is taken from [21] as follows:

$$
\begin{gathered}
y_{1}^{\prime}=-21 y_{1}+19 y_{2}-20 y_{3}, \quad y_{1}(0)=1, \quad 0 \leq x \leq 10, \\
y_{2}^{\prime}=19 y_{1}-21 y_{2}+20 y_{3}, \quad y_{2}(0)=0, \\
y_{3}^{\prime}=40 y_{1}-40 y_{2}-40 y_{3}, \quad y_{3}(0)=-1 .
\end{gathered}
$$


TABLE 3: Numerical results for the nonlinear problem (3).

\begin{tabular}{|c|c|c|c|c|c|}
\hline TOL & Method & IST & FS & TS & MAXE \\
\hline \multirow{4}{*}{$10^{-2}$} & NBDF & 65 & 4 & 69 & $9.38499 e-002$ \\
\hline & VSBBDF & 45 & 0 & 45 & $6.48394 e-004$ \\
\hline & NVSBBDF & 48 & 1 & 49 & $3.53673 e-004$ \\
\hline & VSSBBDF & 49 & 0 & 49 & $3.77477 e-006$ \\
\hline \multirow{4}{*}{$10^{-4}$} & NBDF & 107 & 7 & 114 & $1.02574 e-004$ \\
\hline & VSBBDF & 63 & 0 & 63 & $6.48780 e-005$ \\
\hline & NVSBBDF & 70 & 4 & 74 & $1.59269 e-005$ \\
\hline & VSSBBDF & 80 & 0 & 80 & $1.17305 e-008$ \\
\hline \multirow{4}{*}{$10^{-6}$} & NBDF & 134 & 10 & 144 & $1.03536 e-005$ \\
\hline & VSBBDF & 99 & 0 & 99 & $6.48812 e-006$ \\
\hline & NVSBBDF & 110 & 8 & 118 & $8.11971 e-007$ \\
\hline & VSSBBDF & 169 & 0 & 169 & $2.94571 e-010$ \\
\hline
\end{tabular}

TABLE 4: Numerical results for problem (4).

\begin{tabular}{|c|c|c|c|c|c|}
\hline TOL & Method & IST & FS & TS & MAXE \\
\hline \multirow{4}{*}{$10^{-2}$} & NBDF & 48 & 8 & 56 & $4.92270 e-002$ \\
\hline & VSBBDF & 46 & 0 & 46 & $4.09523 e-003$ \\
\hline & NVSBBDF & 48 & 0 & 48 & $5.56533 e-005$ \\
\hline & VSSBBDF & 50 & 0 & 50 & $5.27731 e-006$ \\
\hline \multirow{4}{*}{$10^{-4}$} & NBDF & 100 & 15 & 115 & $5.96300 e-004$ \\
\hline & VSBBDF & 66 & 0 & 66 & $4.10262 e-004$ \\
\hline & NVSBBDF & 73 & 0 & 73 & $6.47609 e-007$ \\
\hline & VSSBBDF & 96 & 0 & 96 & $7.42231 e-008$ \\
\hline \multirow{4}{*}{$10^{-6}$} & NBDF & 167 & 19 & 186 & $1.04720 e-005$ \\
\hline & VSBBDF & 117 & 1 & 118 & $2.31132 e-006$ \\
\hline & NVSBBDF & 142 & 0 & 142 & $6.61553 e-009$ \\
\hline & VSSBBDF & 224 & 0 & 224 & $7.93169 e-010$ \\
\hline
\end{tabular}

The exact solution is as follows:

$$
\begin{gathered}
y_{1}(x)=0.5\left[e^{-2 x}+e^{-40 x}(\cos 40 x+\sin 40 x)\right], \\
y_{2}(x)=0.5\left[e^{-2 x}-e^{-40 x}(\cos 40 x+\sin 40 x)\right], \\
y_{3}(x)=2 e^{-40 x}\left[-\frac{1}{2} \cos 40 x+\frac{1}{2} \sin 40 x\right] .
\end{gathered}
$$

The eigenvalues are $-2,-40+40 i$, and $-40-40 i$.

\section{Numerical Results}

In the following are tables of results indicating the maximum error and the total steps taken to solve each of the problems given in the previous section. Each problem is solved at a prescribed tolerance, that is, $10^{-2}, 10^{-4}$, and $10^{-6}$. The results are compared with some known successful BDF based algorithms like the nonblock BDF method (Gear's method), the VSBBDF [14] method, and the NVSBBDF [15] method. Also given are the graphs of the performance profile for the methods.
The following notations are used in presenting the results in Tables 1, 2, 3, and 4:

TOL: Tolerance value used

Method: Name of the method

IST: Number of successful steps

FS: Number of failed steps

TS: Total steps

MAXE: Maximum error.

From Tables 1, 2, 3, and 4, the maximum error for the VSSBBDF method is less when compared to the NBDF, VSBBDF, and the NVSBBDF methods. This is also depicted clearly in the graphs shown in Figures 4, 5, 6, and 7. To compare between the methods, we can therefore conclude that the present scheme is more accurate. This is achieved by an optimal choice of the constant $\rho$ that gives better stability region and ensures accuracy at the same time. Furthermore, the total number of steps to complete the integration is competitive for all the problems tested. 


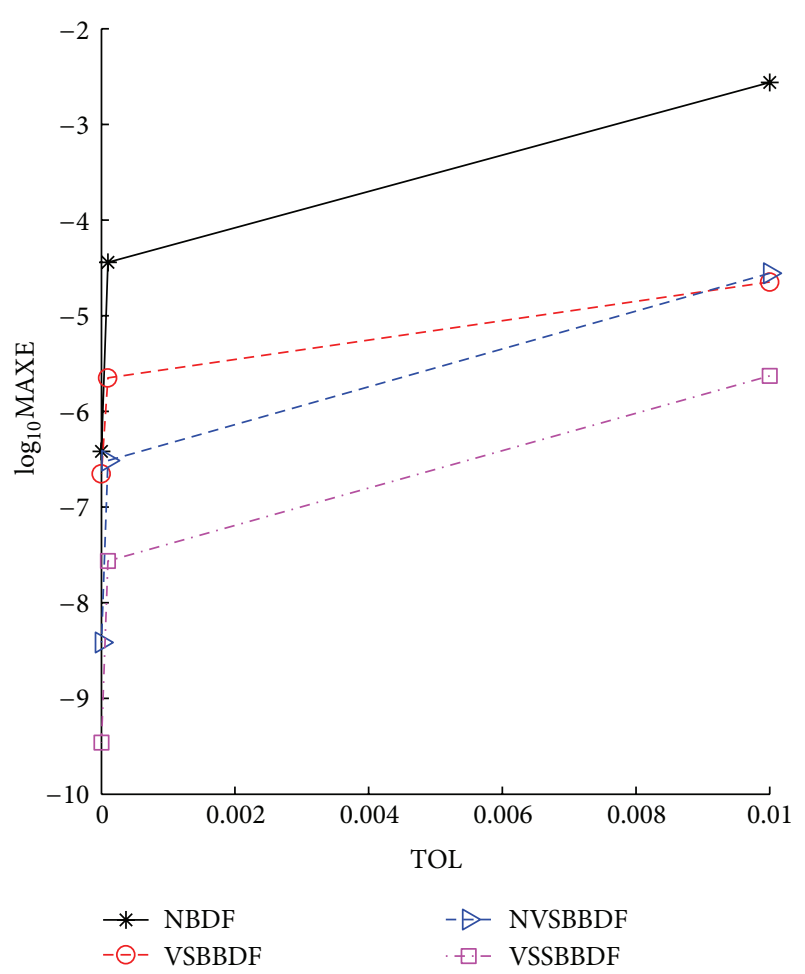

FIGURE 4: Graph of $\log _{10}$ MAXE versus TOL for problem (1).

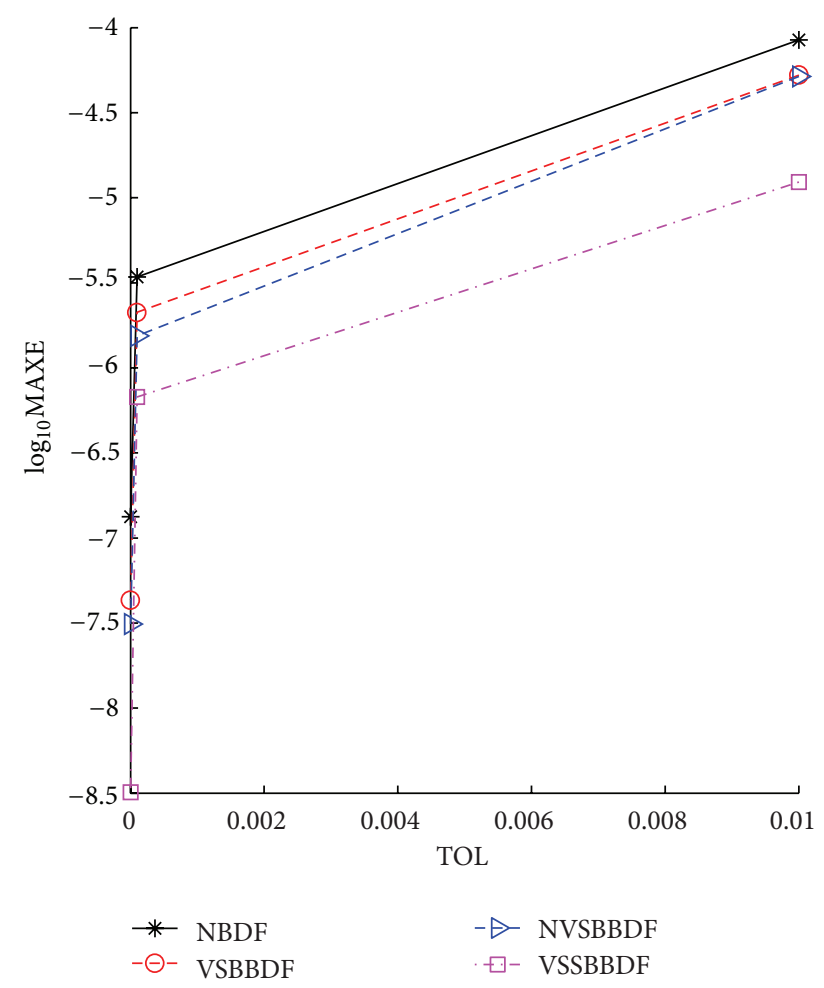

FIgURE 5: Graph of $\log _{10}$ MAXE versus TOL for problem (2).

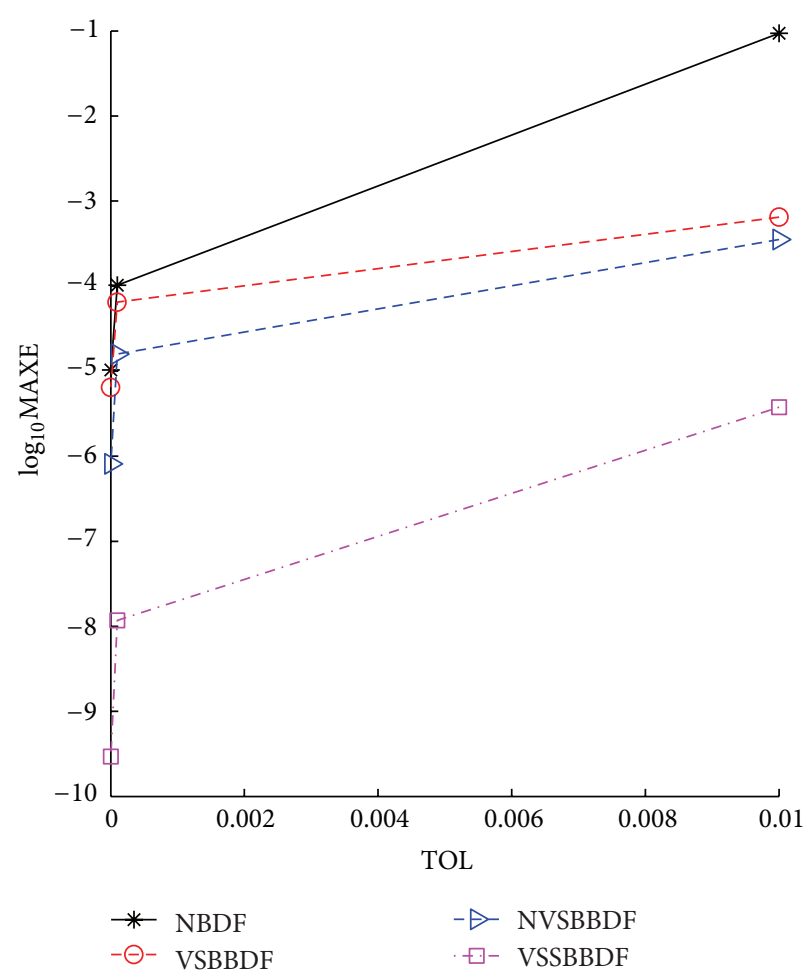

FIgURE 6: Graph of $\log _{10}$ MAXE versus TOL for problem (3).

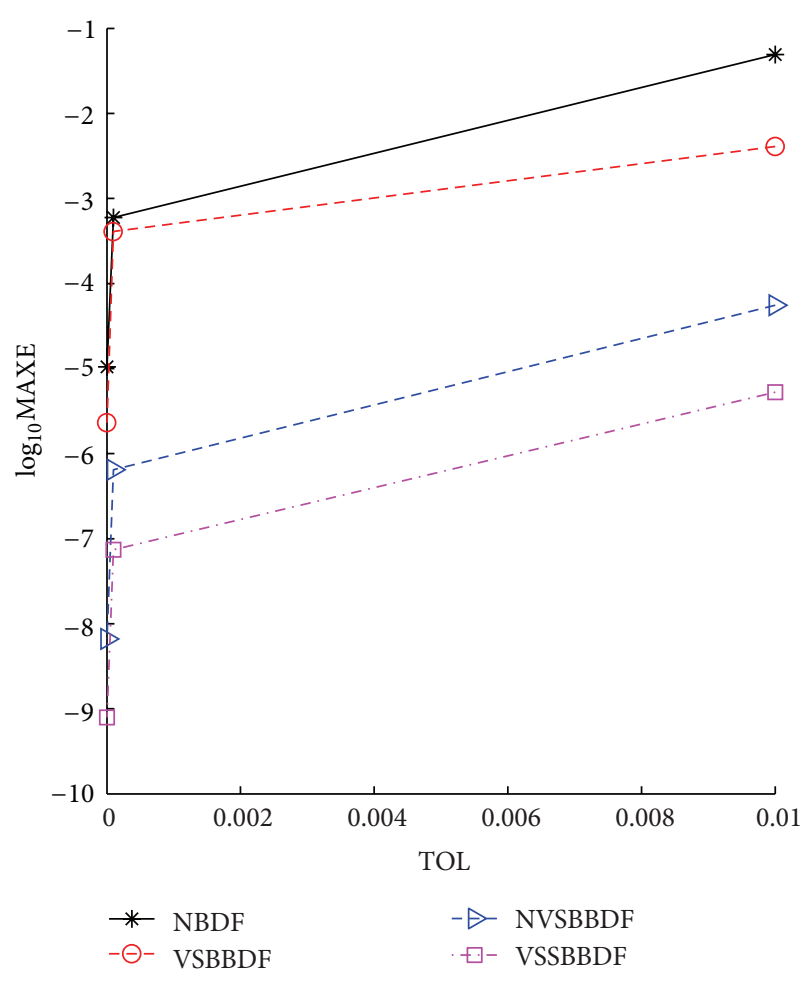

FIGURE 7: Graph of $\log _{10}$ MAXE versus TOL for problem (4). 


\section{Conclusion}

A new variable step size algorithm based on block backward differentiation formula is developed for the integration of stiff problems occurring in the fields of engineering and physical sciences. The step size changing algorithm involves a strategy of increase by a multiple of 1.7, halving, or maintaining the current step size. By choosing $\rho=3 / 8, A$-stable methods are obtained corresponding to the step size ratio of 1 and 2 . This is an added advantage to the method because in most $\mathrm{BDF}$ algorithms, the method is not $A$-stable, but almost $A$-stable when the step ratio is 1 . In addition, choosing $\rho=0$ reduced the algorithm developed to the BBDF, thus making it its superclass. The results obtained show the suitability of the method for solving stiff physical problems and indicate better accuracy when compared with some existing BDF algorithms. Thus, the algorithm developed can be an alternative solver for initial value problems arising in engineering and physical sciences.

\section{Acknowledgments}

The authors thank the anonymous reviewers for their comments which improved the paper and the Institute for Mathematical Research, Universiti Putra Malaysia, for funding the research.

\section{References}

[1] L. Brugnano, F. Mazzia, and D. Trigiante, "Fifty years of stiffness," in Recent Advances in Computational and Applied Mathematics, pp. 1-21, 2011.

[2] J. Álvarez and J. Rojo, "An improved class of generalized RungeKutta methods for stiff problems. I. The scalar case," Applied Mathematics and Computation, vol. 130, no. 2-3, pp. 537-560, 2002.

[3] K. Dekker and J. G. Verwer, Stability of Runge-Kutta Methods for Stiff Nonlinear Differential Equations, vol. 984, North-Holland, Amsterdam, The Netherlands, 1984.

[4] E. Hairer and G. Wanner, Solving Ordinary Differential Equations II: Stiff and Differential-Algebraic Problems, vol. 2, Springer, New York, NY, USA, 2004.

[5] K. Bohmer and H. J. Stetter, Defect Correction Methods: Theory and Applications, vol. 5, Springer, New York, NY, USA, 1984.

[6] R. Frank and C. W. Ueberhuber, "Iterated defect correction for the efficient solution of stiff systems of ordinary differential equations," BIT Numerical Mathematics, vol. 17, no. 2, pp. 146159, 1977.

[7] B. Gustafsson and W. Kress, "Deferred correction methods for initial value problems," BIT Numerical Mathematics, vol. 41, no. 5, pp. 986-995, 2001.

[8] A. T. Layton and M. L. Minion, "Implications of the choice of quadrature nodes for Picard integral deferred corrections methods for ordinary differential equations," BIT Numerical Mathematics, vol. 45, no. 2, pp. 341-373, 2005.

[9] G. G. Dahlquist, "A special stability problem for linear multistep methods," BIT Numerical Mathematics, vol. 3, pp. 27-43, 1963.

[10] J. R. Cash, "On the integration of stiff systems of O.D.E.s using extended backward differentiation formulae," Numerische Mathematik, vol. 34, no. 3, pp. 235-246, 1980.
[11] J. R. Cash, "Modified extended backward differentiation formulae for the numerical solution of stiff initial value problems in ODEs and DAEs," Journal of Computational and Applied Mathematics, vol. 125, no. 1-2, pp. 117-130, 2000.

[12] H. Musa, M. B. Suleiman, and F. Ismail, "A-stable 2-point block extended backward differentiation formula for solving stiff ordinary differential equations," AIP Conference Proceedings, vol. 1450, pp. 254-258, 2012.

[13] Z. B. Ibrahim, K. I. Othman, and M. Suleiman, "Implicit $r$-point block backward differentiation formula for solving first-order stiff ODEs," Applied Mathematics and Computation, vol. 186, no. 1, pp. 558-565, 2007.

[14] Z. B. Ibrahim, M. Suleiman, and K. I. Othman, "Fixed coefficients block backward differentiation formulas for the numerical solution of stiff ordinary differential equations," European Journal of Scientific Research, vol. 21, no. 3, pp. 508-520, 2008.

[15] M. B. Suleiman, H. Musa, F. Ismail, and N. Senu, "A new variable step size block backward differentiation formula for solving stiff IVPs," International Journal of Computer Mathematics, 2013.

[16] J. Williams and F. de Hoog, "A class of $A$-stable advanced multistep methods," Mathematics of Computation, vol. 28, pp. 163177, 1974.

[17] S. A. M. Yatim, Z. B. Ibrahim, K. I. Othman, and M. B. Suleiman, "A quantitative comparison of numerical method for solving stiff ordinary differential equations," Mathematical Problems in Engineering, vol. 2011, Article ID 193691, 12 pages, 2011.

[18] S. A. M. Yatim, Z. B. Ibrahim, K. I. Othman, and M. B. Suleiman, "A numerical algorithm for solving stiff ordinary differential equations," Mathematical Problems in Engineering, vol. 2013, Article ID 989381, 11 pages, 2013.

[19] D. Kushnir and V. Rokhlin, "A highly accurate solver for stiff ordinary differential equations," SIAM Journal on Scientific Computing, vol. 34, no. 3, pp. A1296-A1315, 2012.

[20] H. Aminikhah and M. Hemmatnezhad, "An effective modification of the homotopy perturbation method for stiff systems of ordinary differential equations," Applied Mathematics Letters, vol. 24, no. 9, pp. 1502-1508, 2011.

[21] J. D. Lambert, Computational Methods in Ordinary Differential Equations, John Wiley \& Sons, New York, NY, USA, 1973. 


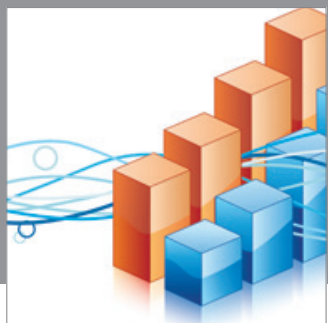

Advances in

Operations Research

mansans

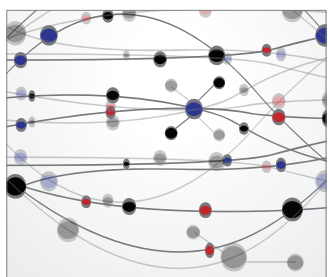

The Scientific World Journal
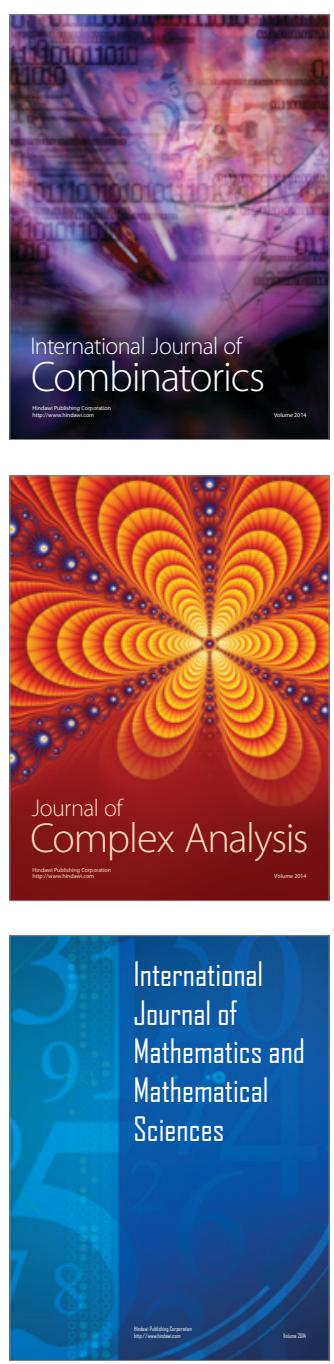
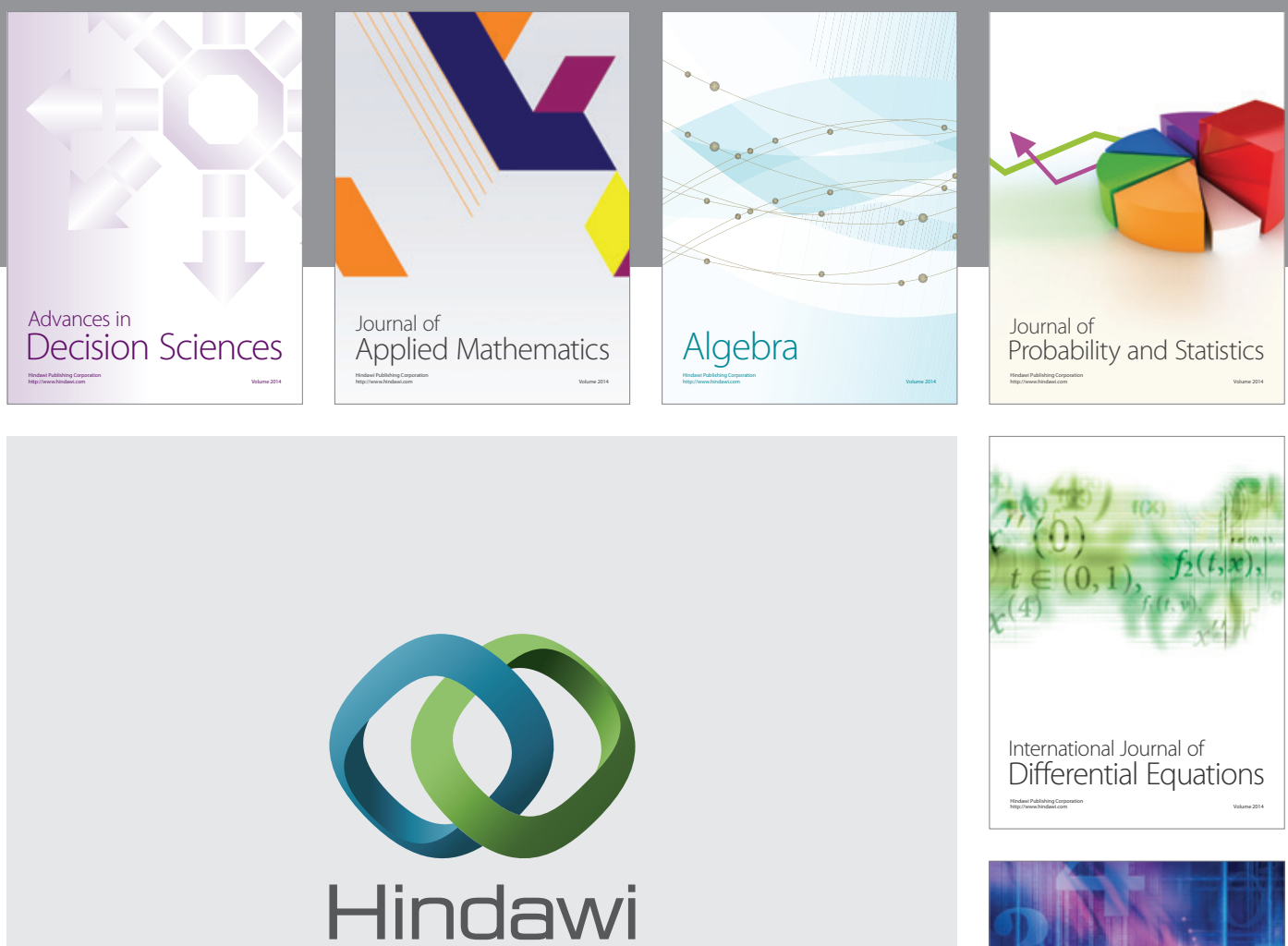

Submit your manuscripts at http://www.hindawi.com
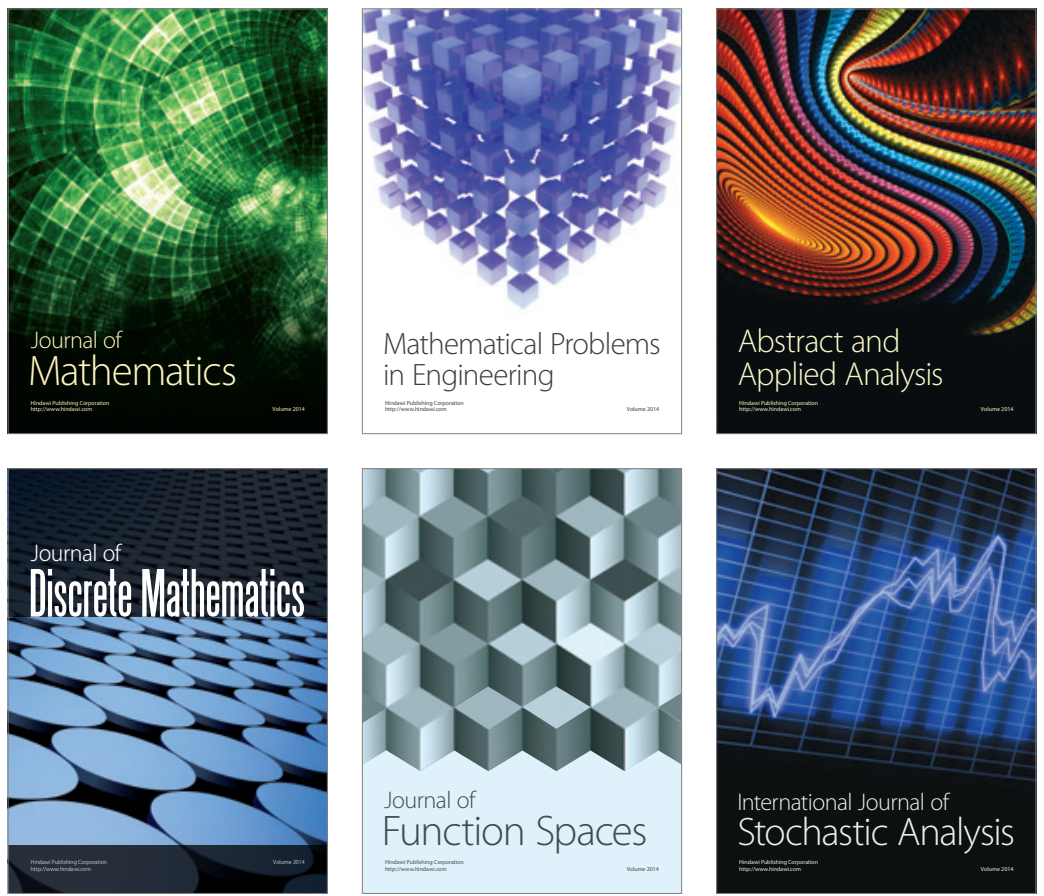

Journal of

Function Spaces

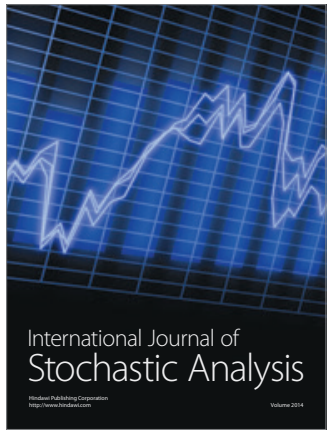

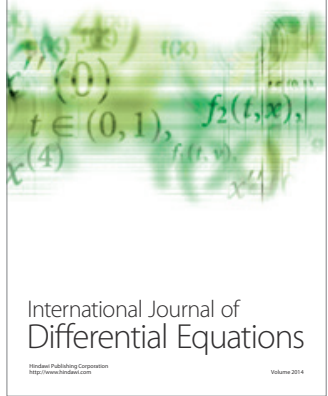
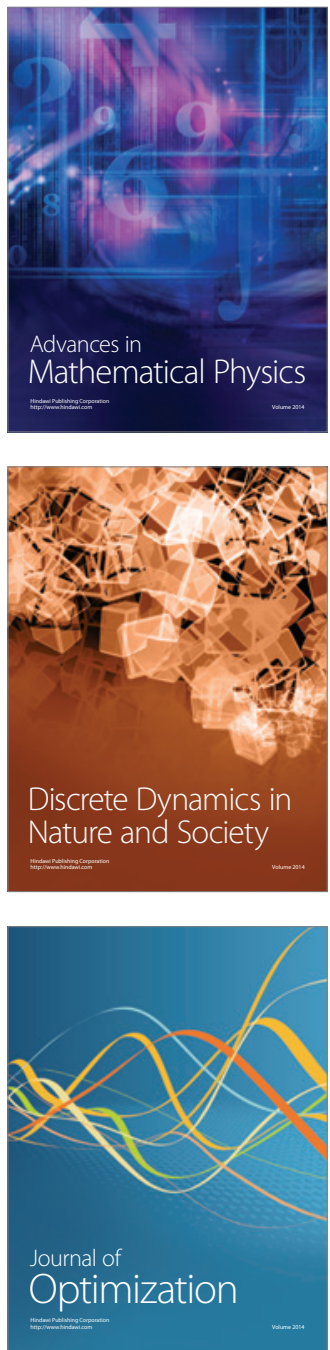